\title{
Saccadic performance characteristics and the behavioural neurology of Tourette's syndrome
}

\author{
Robert H Farber, Neal R Swerdlow, Brett A Clementz
}

\begin{abstract}
Objective-To better understand the neuropathological correlates of Tourette's syndrome (TS), measures of saccadic eye movement performance were examined among patients with TS.

Methods-A case-control design was used. Twenty one patients with DSM-IV TS (mean age 40.6 years (SD 11.0); $38 \%$ female) mainly recruited from UCSD Psychiatry Services, and a community based sample of 21 normal subjects (mean age 34.6 years (SD 13.4); $43 \%$ women) participated in this study. Participants were administered ocular motor tasks assessing visual fixation, and the generation of prosaccades, predictive saccades, and antisaccades. Saccadic reaction time, amplitude, duration, and mean and peak velocity were computed. Intrusive saccades during visual fixation and the proportion of correct antisaccade responses were also evaluated.
\end{abstract}

Results-The groups had similar visual fixation performance. Whereas patients with TS generated prosaccades with normal reaction times and amplitudes, their saccade durations were shorter and their mean velocities were higher than in normal subjects. During a prosaccade gap task, patients with TS exhibited an increased proportion of anticipatory saccades $(\mathrm{RTs}<90)$. The proportion of "express" saccades $(90<\mathrm{RT}<<135)$ did not differ between groups. Patients with TS had fewer correct antisaccade responses than did normal subjects, an effect accounted for by $19 \%$ of the patients. Antisaccade reaction times among patients with TS were increased during an overlap version of the task.

Conclusion-These findings suggest that TS mildly affects the ocular motor control circuitry associated with saccade inhibition.

(7 Neurol Neurosurg Psychiatry 1999;66:305-312)

Psychiatry, University

of California, San

Diego, CA, USA

N R Swerdlow

Correspondence to:

Dr Brett A Clementz,

Department of Psychology,

01099500 Gilman Drive, La

Jolla, CA 92093-0109, USA.

Telephone 001619534

0524; fax 0016195347190 ;

email bclementz@ucsd.edu

Received 3 June 1998 and in revised form

18 August 1998

Accepted 14 September

1998 (orbitofrontal and insular cortices, and parahippocampal gyrus), ${ }^{7-13}$ and perhaps increased metabolic activity in the premotor cortex. ${ }^{7}$ The reported abnormalities in these regions are theoretically consistent with difficulties inhibiting unwanted motor and vocal behaviours. ${ }^{34-16}$

Saccadic eye movement performance has been used to evaluate the functional integrity of corticosubcortical circuitry among several patient populations. ${ }^{17-25}$ For laboratory testing, saccades can be described on a continuum from more "simple" (for example, prosaccades generated to a novel peripheral stimulus) to more "cognitively complex" responses (for example, predictive, memory guided, and antisaccades). ${ }^{18} 222326$ Performance on saccade tasks may be used to evaluate hypotheses about the location(s) of neuropathology. ${ }^{22}{ }^{27-30} \mathrm{Ad}-$ ministering both simple and cognitively complex saccade tasks to patients with TS, therefore, may be helpful for investigating the neurological correlates of this disorder.

Because TS is associated with behavioural disinhibition, saccade tasks assessing this phenomenon may be particularly useful for assessing the adequacy of ocular motor control among patients with this illness. ${ }^{17}{ }^{27}{ }^{30-32}$ Fixation tasks require subjects to maintain gaze at a specified location for a requisite time interval. Saccadic intrusions during fixation may be an index of failed inhibition. ${ }^{19}{ }^{21}$ Excessive anticipatory responses during prosaccade and predictive saccade tasks may also be indices of failed inhibition. ${ }^{32}$ Antisaccade performance is also used to assess inhibitory abilities. ${ }^{23}{ }^{33}$ Correct responses are generated to the mirror locations (same amplitude, opposite direction) of peripheral cues, and antisaccade errors (saccades to the peripheral cues) are typically interpreted as inhibitory failures.

Previous research on eye movement performance among patients with TS is both sparse and difficult to interpret. Lasker et al examined saccadic system functioning among patients with Huntington's disease and patients with TS $(n=8) .{ }^{19}{ }^{20}$ For most analyses, the ocular motor data from patients with TS were pooled with those of normal subjects and subjects with developmental dyslexia. The graphically presented data for a response suppression task in the study of Lasker et al showed patients with TS falling between the normal and Huntington's samples (see their fig $2, \mathrm{p} 367$ ).$^{19}$

Bollen et al reported normal smooth pursuit and saccadic eye movements among 28 children with TS. ${ }^{34}$ This report did not include measures of central tendency or variability, lacked formal statistical analyses, and did not include data from a normal comparison 
sample. In a case study by Narita et al, a 13 year old boy with TS exhibited hypometric saccades. ${ }^{35}$ Additionally, the patient had a marked inability to generate antisaccades compared with a healthy 9 year old subject. Straube et al reported that 10 adult patients with TS had normal prosaccade metrics, but had increased antisaccade latencies, reduced antisaccade peak velocities, and had difficulty generating correct sequences of memory guided saccades. ${ }^{36}$

Previous studies provided a useful background for planning the current investigation. To further evaluate ocular motor behaviour in TS, adult patients and normal subjects were administered tasks assessing visual fixation, and the generation of prosaccades, predictive saccades, and antisaccades. The behavioural symptoms and associated neuropathological theories of TS suggest that these patients may exhibit deficits of saccade inhibition indicative of prefrontal cortex or basal ganglia pathology.

\section{Methods}

SUBJECTS

Twenty one patients (mean age 40.6 (SD 11.0), range $=18-58 ; 38 \%$ women) with DSM-IV TS ${ }^{1}$ and 21 normal comparison subjects (mean age 34.6 (SD 13.4), range $=21-59$; $43 \%$ women) participated in this study. Participants were in good physical health, without neurological signs, not taking anxiolytic or sedative hypnotic drugs, and free from current psychoactive substance use disorders and a personal or family history of psychotic disorders based on self reports. All subjects provided informed consent.

Patients with TS

Patients were recruited from UCSD Psychiatry Services and San Diego chapters of the Tourette Syndrome Association. They were clinically evaluated using a medical history questionnaire, the structured clinical interview for DSM-III-R diagnoses (SCID-P, modules B, C, E, F), ${ }^{37}$ a DSM-III-R and DSM-IV Tourette symptom checklist, the Yale global tic severity scale (YGTSS; global severity score median $=33$, interquartile range $=28-58),{ }^{38}$ and the Hamilton rating scale for depression (HAM-D; median=5, interquartile range= $4-8) .{ }^{39}$ Past or present symptoms of attention deficit hyperactivity disorder were evaluated using the medical history questionnaire. Thirty three per cent (seven of 21) of the patients had experienced at least one symptom associated with either attention deficit or hyperactivity. The Yale-Brown obsessive-compulsive scale (YBOCS) was also administered to $52 \%$ (11 of 21) of the patients who endorsed current obsessive-compulsive symptoms (O-C combined score median $=11$, interquartile range $=7-15) . .^{40}$ None of the patients endorsed any psychotic symptoms. Only 33\% (seven of 21) of the patients were receiving psychotrophic medications at the time of testing (two on fluoxetine; two on sertraline; one on haloperidol; one on clonidine; and one on fluoxetine, perphenazine, clonidine, and clonazepam).
Normal subjects

Normal subjects were recruited from the San Diego community through advertisements. They were evaluated with the Minnesota multiphasic personality inventory (MMPI) ${ }^{41}$ and were screened for a history of psychiatric disorders among their first degree biological relatives. Only subjects without a major affective disorder, a psychotic disorder, an elevation ( T score >70) on MMPI scales L, F, 2, 6, 7, 8, the MacAndrews alcoholism and Wiggins psychoticism scales, a Goldberg index $>60,{ }^{42}$ or a family history of psychotic disorder, suicide, or admission to a psychiatric hospital were asked to participate.

\section{APPARATUS}

Ocular motor data were collected in a quiet, darkened $\left(<0.1 \mathrm{~cd} / \mathrm{m}^{2}\right)$ room. Horizontal eye movements were recorded using an Eye Trak Model 210 eye movement monitor and infrared spectacles (4 ms time constant) mounted on eye glass frames (Applied Science Laboratories, Waltham, MA, USA). The subjects' head position was stabilised using a bite bar. Stimuli were presented on a high resolution Zenith flat surface colour monitor (model ZCM-1792) positioned $43 \mathrm{~cm}$ from the subjects' eyes. Eye movement recordings from both eyes were digitised at $256 \mathrm{~Hz}$ using a Data Translation (DT2821) A to D board connected to an IBM compatible computer. Recordings were displayed on a video screen so performance could be monitored continuously by the experimenter.

\section{PROCEDURE}

Subjects made an impression on dental wax affixed to the bite bar. They put on the infrared spectacles that were secured with an elastic band placed around the head. Subjects were then seated in front of the video monitor and positioned themselves on the bite bar. The background luminance $\left(0.1 \mathrm{~cd} / \mathrm{m}^{2}\right)$, and size $\left(1^{\circ}\right.$ of visual angle, within which was a small central spot subtending a few minutes of arc) and luminance $\left(1.6 \mathrm{~cd} / \mathrm{m}^{2}\right)$ of the stimuli remained constant throughout testing. Before each task, subjects were presented with calibration targets at central fixation and $\pm 5,10,15$, and $17.5^{\circ}$. Order of task presentation was constant and identical to that described below (fig $1)$.

\section{Fixation task}

A trial began with a target presented at the central fixation. Subjects were told to maintain central fixation for the requisite time period (30 s). During fixation, \pm 4 or $8^{\circ}$ distractor stimuli were presented for $100 \mathrm{~ms}$ at pseudorandom time intervals (every 2.5-4.5 s). Three blocks were presented with eight single distractors per block.

\section{Prosaccade, midpoint task}

The target jumped to different amplitudes $( \pm 5$, $10,15,20,25,30$, and $35^{\circ}$; centred on central fixation) with a 1.5-2.0 s intertrial interval. Subjects were told to find the target as quickly and accurately as possible. Four blocks of 28 
A Fix

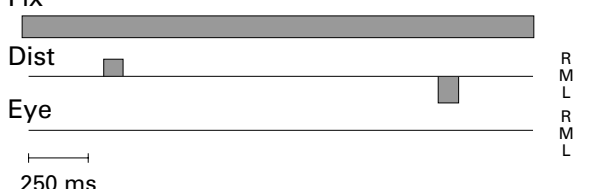

B Fix

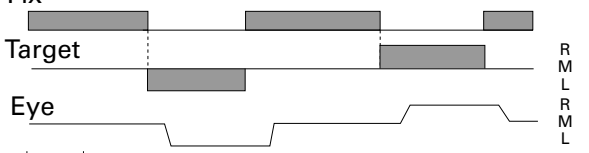

$\stackrel{\longmapsto}{500 \mathrm{~ms}}$

C Fix

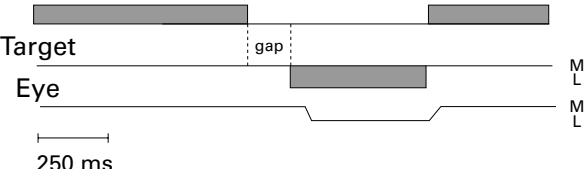

D Target

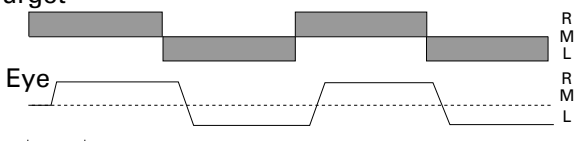

$\stackrel{500 \mathrm{~ms}}{\longrightarrow}$

E Fix

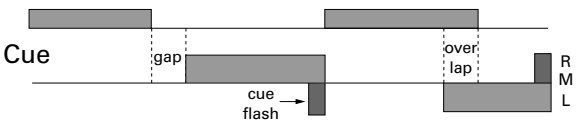

Eye

$$
500 \mathrm{~ms}
$$

Figure 1 Visual representation of the ocular motor paradigms. Fixation point illumination (Fix), target position (Target), and optimal eye position (Eye) tracings are shown. For target and eye position tracings, $M=$ midline, $R=$ right, and $L=$ left. (A) Fixation task: the fixation point remains illuminated during presentation of $100 \mathrm{~ms}$ distractor stimuli (Dist); (B) prosaccade midpoint task: the fixation point is extinguished contemporaneously with the illumination of the peripheral target. Peripheral target duration is $1000 \mathrm{~ms}$; (C) prosaccade gap task: the fixation point is extinguished $200 \mathrm{~ms}$ before the illumination of the peripheral target. Peripheral target duration is $500 \mathrm{~ms} ;$ (D) predictive saccade task: target alternates constantly at 0.4

$\mathrm{Hz}$; (E) antisaccade task: subject is instructed to look to the mirror location of the cue. Cue duration is $1000 \mathrm{~ms} ; A 100$ ms flash is then presented at the cue's mirror location; both $200 \mathrm{~ms}$ gap and $200 \mathrm{~ms}$ overlap conditions are depicted.

trials, with a pseudorandom order of target amplitude presentation, were administered to each subject. The completed task required subjects to generate eight saccades for each amplitude and direction.

Prosaccade, gap task

A trial began with the target at central fixation. After a $1.5-2.0 \mathrm{~s}$ intertrial interval, the target was extinguished. After a 200 ms gap, a peripheral target was illuminated at either $\pm 4^{\circ}$. The target returned to central fixation after $500 \mathrm{~ms}$. Subjects were instructed to find the target as quickly and accurately as possible. Five blocks of 29 trials were presented. Each block contained three "catch" trials during which the peripheral target was not illuminated (the central fixation point reappeared after a 700 ms gap).
Predictive saccade task

The target alternated constantly at $0.4 \mathrm{~Hz}$ between $\pm 5,10$, or $15^{\circ}$ positions. Subjects were told that the target would move in a regular fashion, and they were instructed to keep their eyes on the target as much as possible. Trials were presented for 22 cycles under the three amplitude conditions. Order of presentation for the three conditions was counterbalanced within groups.

\section{Antisaccade task}

A trial began with the target at central fixation. Following a 2.0-2.5 s intertrial interval, the target was extinguished and a cue was illuminated for $1000 \mathrm{~ms}$ at either \pm 8 or $16^{\circ}$ from central fixation. Subjects were instructed to generate a saccade to the equal and opposite location of the cue. After $1 \mathrm{~s}$, a $100 \mathrm{~ms}$ flash appeared at the cue's mirror location to reinforce the accuracy component of the task. The stimulus then returned to central fixation. There were two different fixation conditions: gap and overlap. During gap trials, the illumination of the peripheral cue was preceded by a $200 \mathrm{~ms}$ gap; during overlap trials, the central fixation point remained illuminated for $200 \mathrm{~ms}$ after cue presentation. ${ }^{30}$ A practice block of eight trials was administered to each subject before the test blocks. Four test blocks of 20 trials were presented with a pseudorandom order of cue presentation (10 trials for each cue location, under each condition in each direction).

\section{OCULAR MOTOR ANALYSES}

Waveforms were displayed using ASYST (Version 4.0; Keithley Instruments, Inc). Digitised data were low pass filtered in the frequency domain at $60 \mathrm{~Hz}$. Data from the eye with the cleanest recordings were used for all analyses. For each trial, the position, velocity, and acceleration arrays were presented simultaneously on a high resolution colour monitor. Only trials free of artifacts were scored. Averaging over all tasks, there was a modest difference in the number of useable trials between groups (mean TS 376.4 (SD 44.7); mean normal 417.9 (SD 49.7)). Nevertheless, all subjects had ample data available from which to estimate their "true" performance on all ocular motor tasks.

Our infrared recordings are linear through roughly $\pm 16^{\circ}$; degrees of visual angle/number of digital units is typically a decelerating function for more extreme values (when predicting degrees of visual angle from digital units, the function is sigmoid in shape). To accurately change digital units into degrees of visual angle, we calculated first to fifth order polynomials, and visually inspected their fit to the fixation data. Digitised ocular motor data were then transformed to degrees of visual angle via application of the best fitting function. ${ }^{30-32}$

Each saccadic event was bracketed by the scorer, and reaction time, accuracy, duration, and mean and peak velocity were automatically computed. Saccadic reaction time was defined as the latency (in ms) between target movement and eye velocity increase above $10 \%$ s. Accuracy was measured as saccade amplitude 
in degrees of visual angle. Saccadic duration was defined as the time interval (in $\mathrm{ms}$ ) between eye velocity increase above $10 \%$ and subsequent eye velocity decrease below $10 \%$. Peak velocity was defined as the maximum velocity within the saccade duration window. Only saccades of at least $90 \mathrm{~ms}$ latency were considered stimulus triggered events. ${ }^{28}{ }^{43}$

\section{Fixation task}

For each distractor stimulus, the interval from $250 \mathrm{~ms}$ before to $500 \mathrm{~ms}$ after stimulus presentation was bracketed by the scorer. Saccadic intrusions were identified based on their characteristic position, velocity, and acceleration profiles. ${ }^{22}$ To compare groups, the proportion of usable trials during which a saccadic intrusion occurred was calculated across distractor blocks (six blocks with four distractors each).

Prosaccade tasks

The interval from $250 \mathrm{~ms}$ before to $500 \mathrm{~ms}$ after the target jump was analyased. Prosaccades were defined as the first scorable saccadic event that occurred after fixation point offset.

\section{Predictive saccade task}

The predictive saccade tasks were divided into five successive blocks (four cycles in each block, excluding the first and last cycles). This approach allowed us to evaluate whether subjects learned to predict target relocation over successive trials. Because it is difficult to distinguish small intrusive and corrective saccades from hypometric refixation efforts, only saccades with amplitudes of at least $10 \%$ of the total target excursion were scored (for example, saccades $>3^{\circ}$ of visual angle for the $\pm 15^{\circ}$ condition). ${ }^{32}$ Scorable events were defined as those in the direction of target motion from $1000 \mathrm{~ms}$ before to $1000 \mathrm{~ms}$ after target relocation.

\section{Antisaccade task}

For the antisaccade task, the interval from 250 $\mathrm{ms}$ before to $1000 \mathrm{~ms}$ after cue presentation was analysed. The proportion of useable trials with a correct response (a saccade generated to the opposite screen location of the cue), and the metrics of both correct and error responses (saccades generated to the peripheral cue) were calculated.

\section{Results}

DATA ANALYSES

Data were analysed using mixed design repeated measures analyses of variance (ANOVAs) with Huynh-Feldt adjusted degrees of freedom for the within subjects factors. Helmert contrasts and $t$ tests were used to further examine significant effects. Effect sizes were calculated, when appropriate, by taking the difference between the patient and normal group means and dividing by the SD of the normal group. ${ }^{44}$ Spearman correlations were calculated to examine the relations between saccade and clinical symptom variables.
PRELIMINARY ANALYSES

The groups were similar in age and sex. There were no significant correlations between any of the ocular motor and clinical symptom variables. Patients' medication status was also not correlated with any ocular motor measure. Finally, there were no significant differences on saccade variables between patients with TS with and those without a history of attention deficit hyperactivity symptoms. ${ }^{45-47}$

\section{FIXATION TASK}

The proportion of saccadic intrusions was analysed using a group (TS, normal) by block (1-6) repeated measures ANOVA. The groups did not significantly differ on the proportion of saccadic intrusions across distractor blocks (mean TS 0.04 (SD 0.07), range $=0.00-0.27$; mean normal 0.02 (SD 0.04), range $=0.00-$ 0.14 ; effect size $=0.50$ ). Only a few subjects had intrusive saccades during fixation: $33 \%$ (seven of 21 ) of the patients with TS and $29 \%$ (six of 21 ) of the normal subjects. There were no other significant effects on variables from this task.

PROSACCADE, MIDPOINT TASK

Group by target amplitude $(5,10,15,20,25$, $30,35)$ by direction (left, right) repeated measures ANOVAs were used to evaluate saccadic reaction times, amplitudes, durations, and mean and peak velocities. For saccade duration, there was a significant main effect of group $(F(1,40)=4.59, \mathrm{p}=0.04)$. Patients with TS generated saccades with shorter durations than did normal subjects (mean TS 95.9, (SD 21.9); mean normal 110.5 (SD 22.2); effect size $=-0.66)$. There were no other significant effects on variables from this task.

Main sequence functions were also generated for each group (collapsing over saccade direction) to further evaluate saccade metrics. ${ }^{22}$ Linear $\left(y=a^{\star} x+b\right.$, where $a$ is the slope and $b$ is the $\mathrm{y}$ intercept) and exponential functions $(y=$ $a^{\star}\left[1-\exp ^{(-x / b)}\right]$, where $a$ is the estimated asymptotic value and $b$ is the rate of approach) were fitted to each subjects' data to examine the relations between saccade amplitude and target amplitude (linear); saccade duration and saccade amplitude (linear); and saccade mean and peak velocity and saccade amplitude (exponentials). Weighted least squares regressions were used to estimate parameters. ${ }^{32}$ Consistent with the ANOVA results, the only significant difference was on y intercept values for the saccade duration saccade amplitude relation $(t(40)=2.32, \mathrm{p}=0.03$ (mean TS 46.8 (SD 14.1); mean normal 58.6 ( $\mathrm{SD}=18.7)$; effect size $=-0.63)$.

PROSACCADE, GAP TASK

Saccades were categorised by reaction time as anticipatory (<90 ms), express (90-135 ms), regular (135-250 ms), or long reaction time events $(>250 \mathrm{~ms})$ based on saccadic reaction time distributions from previous publications. ${ }^{28} 294348$ Data were analysed using a group by type (anticipatory, express, regular, long reaction time) by direction (left, right) repeated measures ANOVA. There was a 


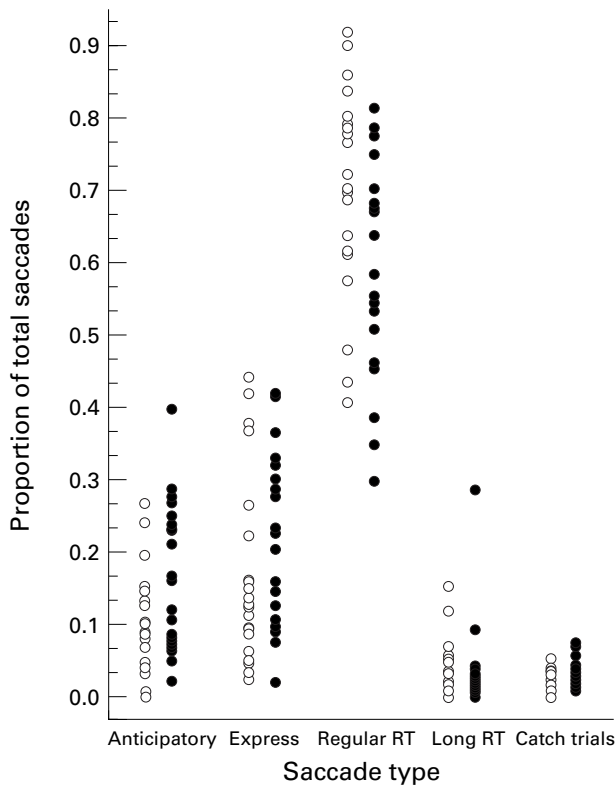

Figure 2 Proportions of anticipatory, express, regular reaction time, long reaction time, and catch trial saccades collapsed across target direction for patients with TS (•) and normal subjects (O).

significant group by saccade type interaction $(F(3,120)=4.04, \mathrm{p}=0.02)$. Patients with TS generated a greater proportion of anticipatory saccades, $t(40)=2.28, \mathrm{p}=0.03$ ) (mean TS 0.16 (SD 0.10); mean normal 0.10 (SD 0.07); effect size $=0.86$ ) and a smaller proportion of regular reaction time saccades than did normal subjects, $t(40)=2.48, \mathrm{p}=0.02$ (mean TS 0.59 (SD 0.15 ); mean normal 0.71 (SD 0.15); effect size $=-0.80)$. The patients with TS also had more saccadic events during the catch trials than did normal subjects, $t(40)=2.85, \mathrm{p}=0.007$ (mean TS 0.032, SD 0.018; mean normal 0.017 , SD 0.016; effect size $=0.94$ ). There were no other significant effects on proportions of saccade types (fig 2).

Group by type by direction ANOVAs were also used to analyse saccade amplitudes, durations, and mean and peak velocities during gap trials. There was a significant main effect of group for saccade duration, $F(1,40)=7.93$, $\mathrm{p}=0.008$. Similar to the midpoint results, patients with TS generated saccades with shorter durations than did normal subjects (mean TS 58.5 (SD 13.4); mean normal 71.9 (SD 17.2); effect size=-0.78). There were no other significant effects for the saccade metrics variables.

PREDICTIVE SACCADE TASK

Predictive saccade variables were analysed using group by block (1-5) by target amplitude $(5,10,15)$ by direction repeated measures ANOVAs. For saccade reaction times, there was a significant main effect of block; $F(4,160)=5.95, \mathrm{p}<0.001$. Subjects had slower reaction times during the first block than during the remaining blocks (fig 3 ). There were no other statistically significant effects for the predictive saccade variables.

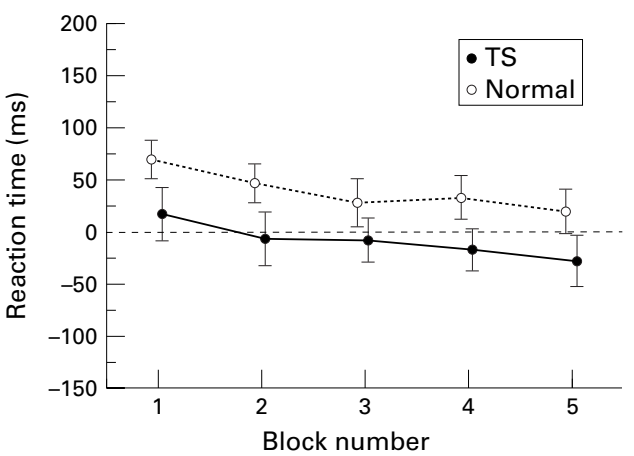

Figure 3 Predictive saccade reaction times (means SEM) collapsed across target amplitude and direction for patients with TS and normal subjects. Block numbers indicate when with TS and normal subjects. Block numbers indicate when
during the course of the task reaction times were averaged. The dashed line at $0 \mathrm{~ms}$ indicates when the new target actually appeared.

ANTISACCADE TASK

The proportion of correct responses and the saccade metrics of correct and error responses were analysed using group by fixation condition (gap, overlap) by target amplitude $(8,16)$ by direction repeated measures ANOVAs. For the proportion of correct antisaccade trials, there was a significant main effect of group, $F(1,40)=8.12, \mathrm{p}=0.007$. Patients with TS had a significantly lower proportion of correct antisaccade responses than did normal subjects (mean TS $0.75(\mathrm{SD}=0.15)$; mean normal 0.86 (SD 0.10); effect size=-1.10; fig 4). There were no other statistically significant effects involving group membership on proportion of correct antisaccade responses.

There was also a significant group by fixation condition interaction on antisaccade reaction times, $F(1,40)=5.11, \mathrm{p}=0.03$. The groups had statistically similar reaction times during gap trials (mean TS 299.9 (SD 52.5); mean normal 282.8 (SD 33.4); effect size $=0.51$ ), but patients with TS had slower reaction times during overlap trials than did normal subjects (mean TS

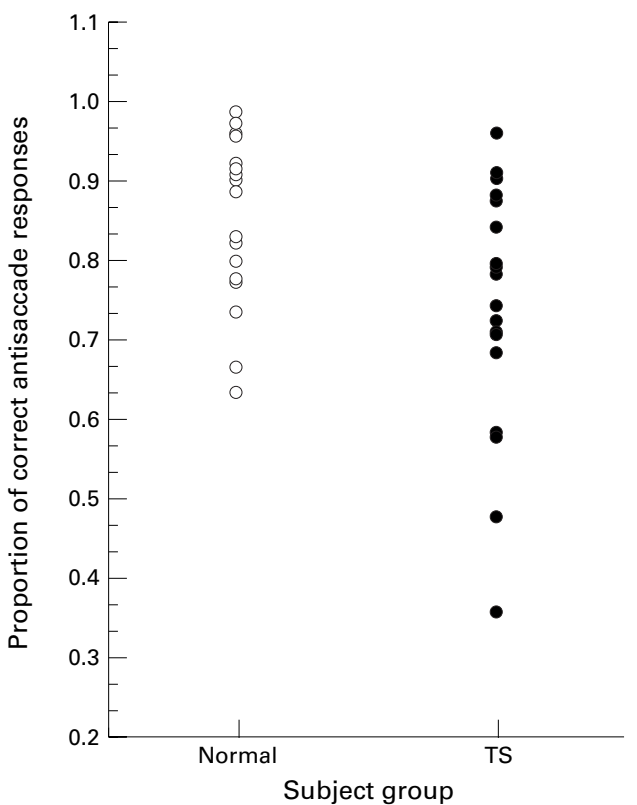

Figure 4 Proportions of correct antisaccade responses collapsed across target type, amplitude, and direction for patients with TS and normal subjects. 


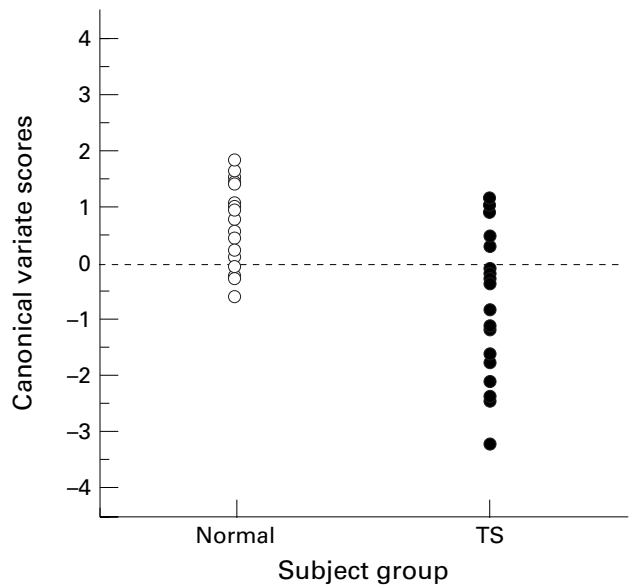

Figure 5 Individual canonical variate scores for patients with TS and normal subjects. The scores represent a linear combination of prosaccade durations, the proportion of anticipatory responses during prosaccade gap trials, and the proportion of correct antisaccade responses.

427.4 (SD 89.1); mean normal 382.4 (SD 53.5); effect size=0.84). There were no other significant effects involving group membership on saccade metrics for the antisaccade variables.

\section{DISCRIMINANT ANALYSES}

An exploratory analysis was used to determine whether a combination of the saccadic measures was superior to any single variable in discriminating TS from normal subjects. Six measures significantly differentiated the groups: (1) midpoint prosaccade duration, (2) gap prosaccade duration, (3) proportion of anticipatory saccades, (4) proportion of catch trial saccades, (5) proportion of correct antisaccades, and (6) correct antisaccade reaction time. Correlations were calculated between these variables. Midpoint prosaccade duration and gap prosaccade duration were highly correlated for both groups (TS $r=0.82$, $\mathrm{p}<0.001$; normal $r=0.96, \mathrm{p}<0.001$ ), as were the proportion of anticipatory saccades and the proportion of catch trial saccades (TS $r=0.57$, $\mathrm{p}=0.007$; normal $r=0.68, \mathrm{p}<0.001)$. Apparently, these pairs of saccadic variables assess similar ocular motor phenomena. Because midpoint prosaccade duration and the proportion of anticipatory saccades were based on larger samples of eye movement behaviour, these variables along with correct antisaccade proportions and reaction times were used for further analysis.

To evaluate the discriminatory power of these four variables in combination, they were entered into a stepwise discriminant analysis (PROC STEPDISC using backward selection; SAS Institute, Inc). With the exception of correct antisaccade reaction time, each variable contributed significantly to the group separation; $F(3,38)=5.80, \mathrm{p}=0.002$ (Partial $R^{2}$ : prosaccade duration $=0.11$; proportion anticipatory saccades $=0.09$; proportion correct antisaccades $=0.12$ ). We then performed a canonical discriminant analysis using these three variables (PROC CANDISC; SAS Institute, Inc). This analytical technique forms a linear combination of variables (a "canonical variate") that maximises group separation. The resulting canonical variate had an effect size of 1.83 , larger than the effect size for any one saccadic variable alone (fig 5; mean canonical variate scores, mean $\mathrm{TS}=-0.66$ (SD 1.22); mean normal 0.66 , SD 0.72).

\section{Discussion}

The present results suggest that TS is associated with a modest difficulty with saccadic inhibition. On average, patients with TS had more anticipatory saccades during prosaccade gap trials and had fewer correct antisaccade responses than did normal subjects. Patients with TS also had shorter saccadic durations across the two prosaccade tasks. In addition, a linear combination of prosaccade durations, proportion of anticipatory saccades during a gap task, and proportion of correct antisaccade responses captured a larger amount of between group variation than any single saccadic variable. These results are consistent with the theory that TS is associated with behavioural disinhibition that is a consequence of dysfunction of basal ganglia thalamocortical circuitry. ${ }^{49} 50$

Saccadic responses are supported subcortically by superior colliculus and basal ganglia structures and cortically by posterior parietal and dorsolateral frontal cortex. ${ }^{22} 26$ The superior colliculus participates in visual fixation and saccade generation. ${ }^{51-54}$ On the one hand, patients with TS had normal proportions of saccadic intrusions during visual fixation and had prosaccades with normal reaction times and amplitudes, so it is unlikely that this disorder is associated with gross impairment of the superior colliculus. On the other hand, patients with TS had shorter duration saccades than did normal subjects. As expected based on the duration results, patients with TS also had higher mean saccade velocities than normal, although this effect was not significant (mean TS 176.3 (SD 32.0); mean normal=157.8 (SD 37.0); effect size $=0.50$ ). Shorter saccade durations and higher saccade mean velocities are found among non-human primates after pharmacological deactivation of collicular fixation cells. $^{53}$ The neurophysiological correlates of this behavioural effect among patients with TS are uncertain. Deactivation of fixation cells tends to disinhibit their collicular burst cell afferents, so perhaps the input to the former is aberrant in TS. Additional research carefully examining the duration characteristics of prosaccades among patients with TS will be necessary to better understand this phenomenon.

Dorsolateral frontal cortex circuitry (including both prefrontal cortex and frontal eye fields) plays an important part in generating cognitively complex saccades (for example, predictive and antisaccades). ${ }^{185}$ Performance during gap, predictive, and antisaccade tasks has been useful for examining the integrity of regions of the prefrontal cortex. ${ }^{1733} 48$ 56-58 Dysfunction of frontal eye fields is associated with decreased proportions of express saccades, decreased proportions of anticipatory saccades, and an inability to reduce saccadic reaction times 
across predictive saccade trials. ${ }^{48}{ }^{57-59}$ Patients with TS, however, generated a normal proportion of express saccades, had an increased proportion of anticipatory responses during gap prosaccade trials, and were able to decrease their reaction times across predictive saccade trials. These findings are inconsistent with dysfunction of frontal eye fields in TS.

Dysfunction of dorsolateral prefrontal cortex circuitry results in fewer correct antisaccade responses ${ }^{17} 19263356$ and perhaps an increased frequency of express saccades during gap tasks. ${ }^{48}$ A small subgroup of patients with TS $(19 \%)$ had fewer correct antisaccade responses than did normal subjects and the groups did not differ on frequency of express saccades. These results would seem to be inconsistent, as a general rule, with prefrontal cortex dysfunction in TS.

There is evidence that TS is associated with mild dysfunction of ocular motor control. The pattern of findings, however, may be inconsistent with involvement of the cortical parts of this neural circuitry. Perhaps short duration saccades, an increased frequency of saccades generated prior to stimulus presentation (anticipatory events), and a modest decrease in proportion of correct antisaccade responses indicate a subcortical dysfunction of the prefrontal cortex circuitry. Additional neurophysiological and neurological studies reporting patterns of saccadic performance among subjects with subcortical dysfunction will be needed to more adequately address this possibility.

There are two additional details to consider when evaluating these data. Firstly, the ocular motor results among patients with TS were not related to medication status. Although few patients were receiving psychotrophic medications at the time of testing $(33 \%)$, their saccadic performance during individual tasks was unremarkable within the TS group. Secondly, both attention deficit and obsessivecompulsive symptoms are found in $\mathrm{TS}^{3}$ Recent studies suggest that the presence of comorbid conditions may adversely affect the performance of patients with TS during neurocognitive and neuromotor tasks. ${ }^{45-47}$ Whereas the assessment of comorbid attention deficit hyperactivity symptoms among our patients with TS was based on self report, the saccadic performance of these patients was not differentiable from that of the patients with TS without a history of inattention or hyperactivity. Prospective studies assessing ocular motor performance among attention deficit hyperactivity disorder patients would be useful for evaluating the specific impact of these symptoms on saccadic response.

This work was supported by grants from the United States Public Health Service (MH49906, MH42228, MH48381, MH01436) and the Tourette Syndrome Association.

1 American Psychiatric Association. Diagnostic and statistical manual of mental disorders. 4th ed. Washington, DC: Amerimanual of mental disorders. 4th

2 Ames D, Cummings JL, Wirshing WC, et al. Repetitive and compulsive behavior in frontal lobe degenerations. $\mathcal{f}$ Psychiatry Clin Neurosci 1994;6:100-13.
3 Cohen DJ, Leckman JF. Developmental psychopathology and neurobiology of Tourette's syndrome. $₹$ Am Acad Child adolesc Psychiatry 1994;33:2-15.

4 Laplane D, Levasseur M, Pillon B, et al. Obsessivecompulsive and other behavioural changes with bilateral basal ganglia lesions: a neuropsychological, magnetic resonance imaging and positron tomography study. Brain 1989;112:699-725.

5 Singer HS. Pathobiology. In: Kurlan R ed. Handbook of Tourette syndrome and related tic and behavioral disorders. Rochester, NY: Marcel Decker, 1993.

6 Singer HS. Neurobiological issues in Tourette syndrome. Brain Dev 1994;16:353-64.

7 Braun AR, Stoetter B, Randolph C, et al. The functional anatomy of Tourette's syndrome: an FDG-PET study. I. Regional changes in cerebral glucose metabolism differentiating patients and controls. Neuropsychopharmacology 1993;9:277-91.

8 Braun AR, Randolph C, Stoetter B, et al. The functional neuroanatomy of Tourette's syndrome: an FDG-PET
study. II: Relationships between regional cerebral metabolism and associated behavioral and cognitive features of the lism and associated behavioral and cognitive features

9 Chase, TN, Geoffrey V, Gillespie M, et al. Structural and functional studies of Gilles de la Tourette syndrome. Rev Neurol 1986;142:851-5.

10 George MS, Trimble MR, Costa DC, et al. Elevated frontal cerebral blood flow in Gilles de la Tourette syndrome: a ${ }^{99 m}$ Tc-HMPAO SPECT study. Psychiatry Res Neuroimaging 1992;45:143-51.

11 Malison RT, McDougle CJ, van Dyck CH, et al. [ $\left.{ }^{123} \mathrm{I}\right] \mathrm{CIT}$ SPECT imaging of striatal dopamine transporter binding in Tourette's disorder. Am F Psychiatry 1995;152:1359-61.

12 Moriarty J, Costa DC, Schmitz B, et al. Brain perfusion abnormalities in Gilles de la Tourette's syndrome. Br f Psychiatry 1995; 167:249-54.

13 Riddle MA, Rasmusson AM, Woods SW, et al. SPECT imaging of cerebral blood flow in Tourette syndrome. Adv Neurol 1992;58:207-11.

14 Baxter LR, Guze, BH. Neuroimaging. In: Kurlan R. Handbook of Tourette syndrome and related tic and behavioral disorders. Rochester: Marcel Decker, 1993.

15 Georgiou N, Bradshaw JL, Phillips JG, et al. The Simon effect and attention deficits in Gilles de la Tourette syndrome and Huntington's disease. Brain 1995;118:1305-18.

16 Baron-Cohen S, Cross P, Crowson M, et al. Can children with Gilles de la Tourette syndrome edit their intentions? Psychol Med 1994;24:29-40.

17 Fukushima J, Fukushima K, Miyasaki K, et al. Voluntary control of saccadic eye movement in patients with frontal cortical lesions and Parkinsonian patients in comparison with that in schizophrenics. Biol Psychiatry 1994;36:21-30.

18 Kennard C, Lueck CJ. Oculomotor abnormalities in Kennard C, Lueck CJ. Oculomotor abnormalities in
diseases of the basal ganglia. Rev Neurol 1989;145:587-95. 9 Lasker AG, Zee DS, Hain TC, et al. Saccades in Huntington's disease: Initiation defects and distractibility. Neurology 1987;37:364-70.

20 Lasker AG, Zee DS, Hain TC, et al. Saccades in Huntington's disease: slowing and dysmetria. Neurology 1988;38:427-31

21 Leigh RJ, Newman SA, Folstein SE, et al. Abnormal ocular motor control in Huntington's disease. Neurology 1983;33: $1268-75$.

22 Leigh RJ, Zee DS. The neurology of eye movements. 2nd ed. Philadelphia: FA Davis, 1991.

23 Pierrot-Deseilligny C. Saccade and smooth-pursuit impairment after cerebral hemispheric lesions. Eur Neurol 1994;34:121-34

24 Rascol O, Clanet M, Montastruc JL, et al. Abnormal ocular movements in Parkinson's disease. Brain 1989;112:1193-

25 Tian JR, Zee DS, Lasker AG, et al. Saccades in Huntington's disease: predictive tracking and interaction between release of fixation and initiation of saccades. Neurology 1991;41: $875-81$

26 Pierrot-Deseilligny C. Cortical control of saccades in man. Acta Neurol Belg 1991;91:63-79.

27 Pierrot-Deseilligny C, Gaymard B, Muri R, et al. Cerebral ocular motor signs. $\mathcal{F}$ Neurol 1997;244:65-70.

28 Fischer B, Weber H, Biscaldi M, et al. Separate populations of visually guided saccades in humans: reaction times and amplitudes. Exp Brain Res 1993;92:528-41.

9 Fischer B, Weber H. Express saccades and visual attention. Behav Brain Sci 1993;16:553-610.

$30 \mathrm{McD}$ owell JE, Clementz BA. The effect of fixation manipulations on antisaccade performance in schizophrenia: studies of diagnostic specificity. Exp Brain Res 1997;115:333 44

31 McDowell JE, Clementz BA. Ocular-motor delayedresponse task performance among schizophrenia patients. Neuropsychobiology 1996;34:67-71.

32 McDowell JE, Clementz BA, Wixted JT. Timing and amplitude of saccades during predictive saccadic tracking in schizophrenia. Psychophys 1996;33:93-101.

33 Guitton D, Buchtel HA, Douglas RM. Frontal lobe lesions in man cause difficulties in suppressing reflexive glances and in generating goal directed saccades. Exp Brain Res 1985;58:455-72.

34 Bollen EL, Roos RAC, Cohen AP, et al. Oculomotor control in Gilles de la Tourette syndrome. F Neurol Neurosurg Psychiatry 1988;51:1081-3.

35 Narita AS, Shawkat FS, Lask B, et al. Eye movement abnormalities in a case of Tourette syndrome. Dev Med Child Neurol 1997;39:270-3. 
36 Straube AS, Mennicken J-B, Riedel M, et al. Saccades in Gilles de la Tourette's syndrome. Mov Disord 1997:12:53646.

37 Spitzer RL, Williams JB, Gibbon M, et al. Structured clinical interview for DSM-III-R. New York: Biometrics Research Department, New York State Psychiatric Institute, 1988.

38 Leckman JF, Riddle MA, Hardin MT, et al. The Yale global tic severity scale: initial testing of a clinician-rated scale of tic severity. $\mathcal{F}$ Am Acad Child Adolesc Psychiatry 1989;28: 566-73.

39 Hamilton M. Development of a rating scale for primary depressive illness. British fournal of Social and Clinical Psychology 1967;6:229-31.

40 Goodman WK, Price LH, Rasmusssen SA, et al. The YaleBrown obsessive-compulsive scale: I. Development, use and reliability. Arch Gen Psychiatry 1989;46:1006-11.

41 Hathaway SR, McKinley JC. Minnesota multiphasic personality inventory. Minneapolis: University of Minnesota, 1982.

42 Butler RN, Jenkins MA, Braff DL. On the abnormality of comparison groups: the identification of psychosiscomparison groups: the identification of psychosisproneness and substance abuse in putatively norn

43 Clementz BA. The ability to produce short reaction time saccades as a function of gap interval among schizophrenia patients Psyon

44 Smith ML, Glass GV, Miller TI. The benefits of psychotherapy. Baltimore: Johns Hopkins University, 1980.

45 Shucard DW, Benedict RHB, Tekoc-Kilic A, et al. Slowed reaction time during a continuous performance test in children with Tourette's syndrome. Neuropsychology 1997;11 $1-9$.

46 Schuerholz LJ, Cutting L, Mazzocco MM, et al. Neuromotor functioning in children with Tourette syndrome with and without attention deficit hyperactivity disorder. $\mathcal{F}$ Child Neurol 1997;12:432-42.

47 Schuerholz LJ, Baumgardner TL, Singer HS, et al. Neuropsychological status of children with Tourette's syndrome with and without attention deficit hyperactivity disdrome with and without attention
48 Braun $\mathrm{D}$, Weber $\mathrm{H}$, Mergner $\mathrm{TH}$, et al. Saccadic reaction times in patients with frontal and parietal lesions. Brain 1992;115:1359-86.

49 Alexander GE, DeLong MR, Strick PL. Parallel organization of functionally segregated circuits linking basal ganglia and cortex [review]. Annu Rev Neurosci 1986;9:357-81.

50 Alexander GE, Crutcher MD, DeLong MR. Basal gangliathalamocortical circuits: parallel substrates for motor, oculomotor, prefrontal and limbic functions [review]. Prog Brain Res 1990;85:119-46.

51 Hikosaka O, Wurtz RH. The basal ganglia. In: Wurtz RH, Goldberg ME, eds. The neurobiology of saccadic eye movements. Amsterdam: Elsevier Press, 1989.

52 Munoz DP, Wurtz RH. Fixation cells in monkey superior colliculus I. Characteristics of cell discharge. $\mathcal{F}$ Neurophysiol 1993;70:559-75.

53 Munoz DP, Wurtz RH. Fixation cells in monkey superior colliculus II. Reversible activation and deactivation. $\mathcal{F} \mathrm{Neu}$ rophysiol 1993;70:576-89.

54 Wurtz RH, Optican LM. Superior colliculus cell types and models of saccade generation. Curr Opin Neurobiol 1994;4: 857-61.

55 Hikosaka O. Role of the forebrain in oculomotor function. Prog Brain Res 1991;87:101-7.

56 Pierrot-Deseilligny C, Rivaud S, Gaymard B, et al. Cortical control of reflexive visually-guided saccades. Brain 1991; 114:1473-85.

57 Rivaud S, Muri RM, Gaymard B, et al. Eye movement disorders after frontal eye field lesions in humans. Exp Brain Res 1994;102:110-20.

58 Sharpe JA. Adaptation to frontal lobe lesions. In: Keller EL, Zee DS, eds. Adaptive processes in visual and oculomotor systems. Oxford: Pergamon Press, 1986:239-46.

59 Bruce CJ, Borden JA. The primate frontal eye fields are necessary for predictive saccadic tracking [abstract]. Soc Neurosci Abs 1986;12:1086. 